\title{
Comparative Performance Evaluation of Routing Algorithms in IEEE 802.11 Ad Hoc Networks
}

\author{
Evaggelos Chatzistavros $^{1}$, Georgios Stamatellos ${ }^{2}$ \\ ${ }^{1}$ Democrius University if Thrace \\ Xanthi, 67100, Greece \\ ${ }^{2}$ Democrius University if Thrace \\ Xanthi, 67100, Greece
}

\begin{abstract}
In this paper we examine the behavior of Ad Hoc networks through simulations, using different routing protocols and various topologies. We examine the difference in performance, using CBR application, with packets of different size through a variety of topologies, showing the impact node placement has on networks performance. We show that the choice of routing protocol plays an important role on network's performance. We also quantify node mobility effects, by looking into both static and fully mobile configurations. Our paper presents a systematic analysis of a variety of different ad hoc network topologies in terms of node placement, node mobility and routing protocols through several simulated scenarios.
\end{abstract}

Keywords: Ad Hoc Networks, DBF , DSR, Mesh Networks, Routing protocols, ZRP.

\section{Introduction}

Ad Hoc networks' advantage is the promise of infrastructure - free communication. In an Ad hoc network configuration, nodes need to cooperate with each other in establishing transmission paths through the network, using the limited capacity and available resources the best possible way.

Network topology can change rapidly when nodes move in a wireless environment. Therefore, it is very likely that packets must be forwarded through different paths/routes every time. Ad hoc routing protocols are used to discover routes between source and destination nodes. They belong in three categories, proactive, reactive and hybrid. In proactive routing protocols [1], nodes maintain routing information to every other node of the network, which is stored in routing tables, which are periodically updated when topology changes. In our simulations we have used DBF (Distributed Bellman Ford), however there are several proactive routing protocols, such as DSDV, GSR, OLSR [1] et.al. In Reactive routing, routes are defined and maintained only for nodes which have data to transmit. Route discovery is performed by sending route discovery packets to the network.

When a node with a route to the destination is found (or the destination itself), a route acknowledgment packet is sent back to the sender. DSR (Dynamic Source Routing), used in our simulations, is a reactive routing protocol. Other reactive protocols are AODV, LMR, TORA [1] et.al. Hybrid routing protocols belong to a newer family of routing protocols, which combine the characteristics of proactive and reactive protocols. Their purpose is to increase scaling, allowing neighboring nodes to cooperate in order to create a backbone and to reduce overhead due to routing discovery, using the most appropriate nodes for route discovery. We used ZRP (Zone Routing Protocol) in our simulations as a representative of hybrid routing protocols from a family of protocols that also includes ZHLS, DDR et.al. [1].

Previous work in performance evaluation of routing protocols is reported in references [2-8]. In [2] and [3] DSR and AODV routing protocols are compared in different scenarios in terms of mobility and offered data load. STAR and DSVD, which are proactive routing protocols, are compared in [4] and [5] respectively, with DSR and AODV, which are reactive routing protocols. The authors of [6] and [7] compare their implementations of DSDV, TORA, DSR and AODV. In [8] reactive routing protocols AODV, PAODV, CBRP, DSR are compared with proactive protocol DSDV and the authors conclude that the four reactive protocols perform better than DSDV. In this paper we compare proactive, reactive and hybrid routing protocols representatives, DBF, DSR and ZRP respectively, through a variety of simulation scenarios, involving both static and fully mobile node topologies. Through this paper we assume the use of IEEE 802.11 Distributed Coordination Function CSMA/CA as the multiple access scheme for the Ad hoc mode. We focus on mobility effects and comparative performance evaluation of routing protocols in Ad Hoc networks. We have 
conducted several simulations involving different network topologies and data load conditions for the examined routing protocols. In sections 2 and 3 we present simulation results on fixed topology networks and networks with limited mobility, respectively. In section 4 we introduce a full mobility random network topology, in section 5 we alter nodes' buffer size and finally in section 6 we present our conclusions.

\section{Fixed Topology Networks}

\subsection{Simulations on Chains of Nodes}

For our simulations, we have used the Qualnet Simulator [9]. Our first scenario involves chains of nodes, whose length increases in each simulation. Nodes are static, using IEEE $802.11 \mathrm{~b}$ and DBF as routing protocol. Node 1 is the source node, transmitting at $2 \mathrm{Mbps}$ with a constant bit rate. The last node of the chain is the destination node (node 6 in Fig. 1), and the intermediate nodes are used only to forward packets.

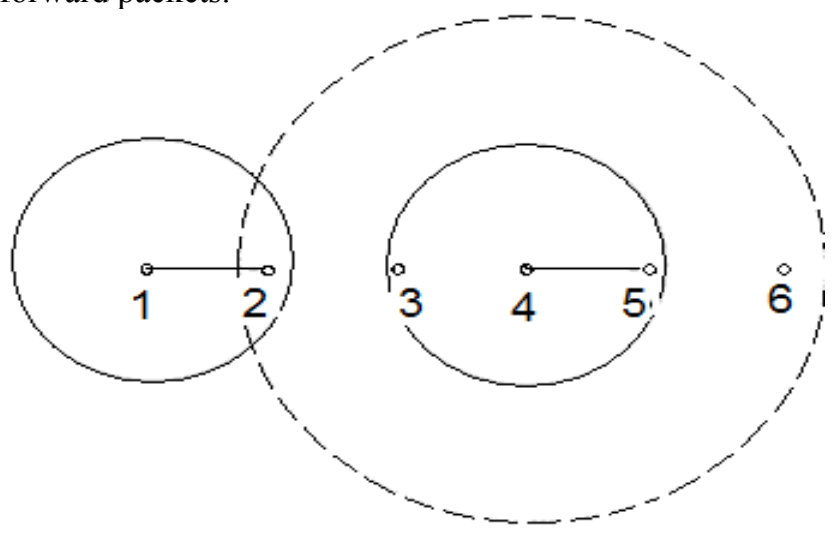

Fig 1: Interference between nodes. Solid and discontinuous circle show transmission and interference range respectively

Each node has a transmission and an interference range shown in Fig. 1 with the solid and the discontinuous circles respectively. For example, packet transmission from node 4 interferes with RTS packets sent by node 1 to node 2. As a result, node 2 either does not receive correctly node 1's packets or cannot send the corresponding CTS, leading to decreased channel utilization. In Fig. 2, throughput decreases as chain length increases, and drops to a minimum of $0.24 \mathrm{Mbps}$ for 1500 bytes packets, because a node's ability to send packets is affected by the existing contention conditions caused by neighboring nodes.

We have conducted the same simulations using DSR and ZRP as well. However we do not present simulation results, as there is no significant difference from the results presented in Fig. 2 and 3 (with DBF).

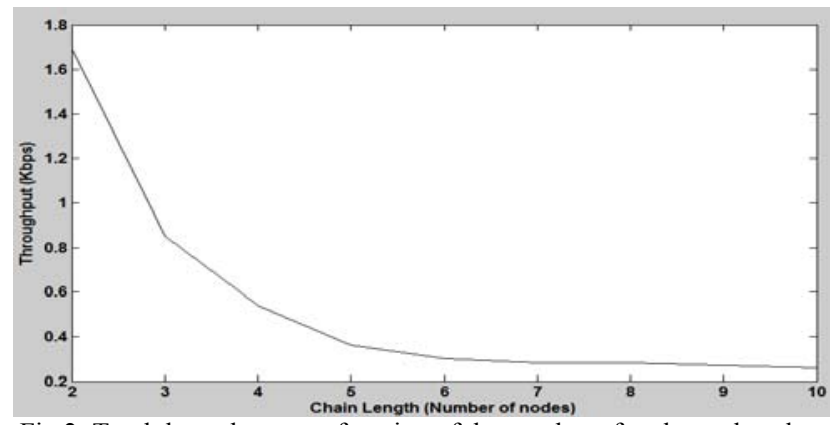

Fig 2: Total throughput as a function of the number of nodes and packet size.

Figure 3 shows the results of another simulation involving the same topology, in which 1500-bytes packets are transmitted through an 8-node chain at different packet rates. Maximum throughput is about $0.4 \mathrm{Mbps}$; however, when the offered traffic load becomes even a little higher than this value, actual throughput drops considerably.

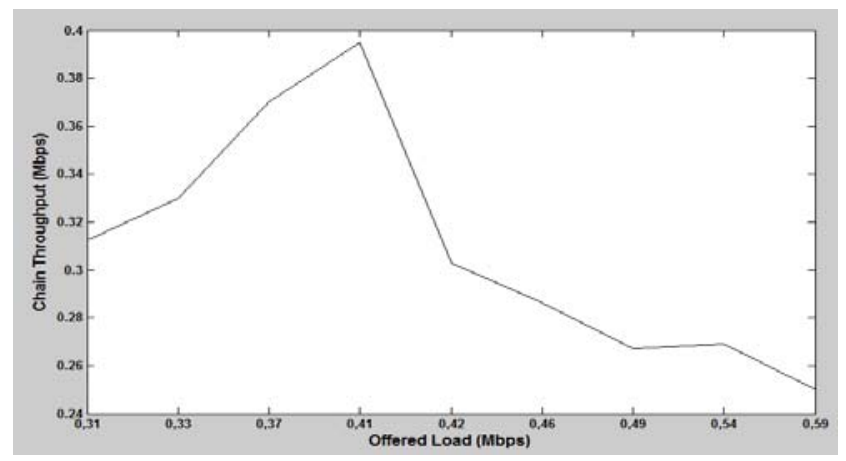

Fig 3: Chain throughput as a function of the offered load

\subsection{Simulations in Square Lattice Networks}

In this section we examine the performance of a mesh network [10] (with fixed nodes as shown in Fig.4), which consists of parallel chains in which data transmission is horizontal. The number of nodes per chain is equal to the number of chains consisting the network.

As mentioned before, network size varies in each simulation between a network which consists of three horizontal chains with three nodes each ( $3 \times 3$ network) and a network which consists of 10 horizontal chains of nodes with ten nodes each (10x10 network). Nodes have a $200 \mathrm{~m}$ distance from their neighbors. For each topology, there are three different scenarios, using different routing protocols: DBF, DSR or ZRP. For each of these routing protocols, we run three simulations, changing CBR packets' size. We use three different packet sizes, 64, 500 and 1500 bytes. Nodes send at a fixed rate of 40 packets/sec. All nodes are static, and use $11 \mathrm{Mbps} 802.11$ Distributed Coordination Function CSMA/CA as the MAC Layer protocol. 


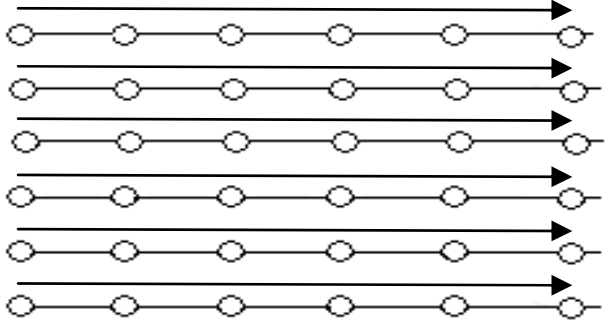

Fig. 4: Lattice network of 6 chains of nodes with 6 nodes each and 6 horizontal flows

Simulation parameters are presented in Table 1.

Table 1: Simulation Parameters

\begin{tabular}{|l|r|}
\hline Protocols & DBF,DSR,ZRP \\
\hline Simulation time & $600 \mathrm{sec}$ \\
\hline Number of nodes & 9 to 100 \\
\hline Simulation area & $2000 \times 2000$ \\
\hline Traffic Type & Constant bit rate \\
\hline Packet Size & $64,500,1500$ bytes \\
\hline Offered load & 40 packets $/ \mathrm{sec}$ \\
\hline Number of connections & 3 to 10 \\
\hline
\end{tabular}

In Figure 5, it is shown that as the network size increases, overall throughput is stabilized approximately at $0.1 \mathrm{Mbps}$, for 1500 bytes packets, which is a value slightly smaller than the one estimated theoretically in [11].

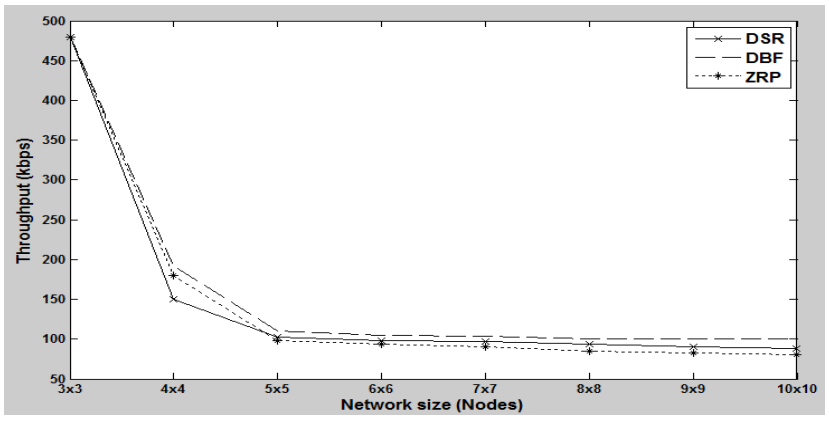

Fig 5: Average per flow throughput in square lattice network as a function of network size and routing protocol for 1500 bytes packets

Figures 6 and 7 show average throughput for 500 and 64 bytes packets respectively.

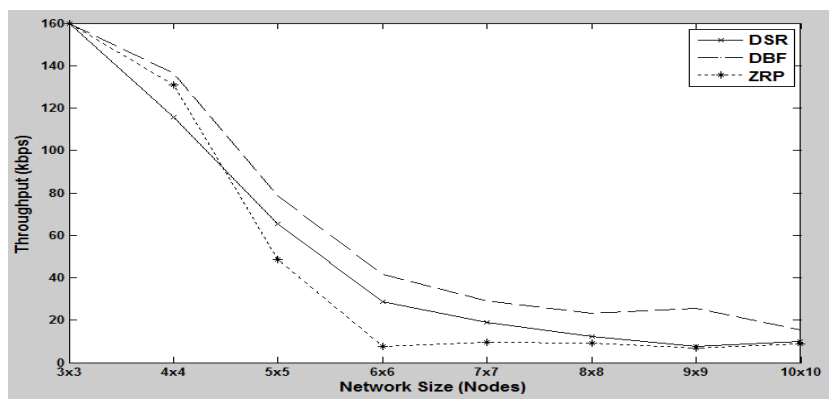

Fig 6: Average per flow throughput in square lattice network, as a function of network size and routing protocol for 500 bytes packets

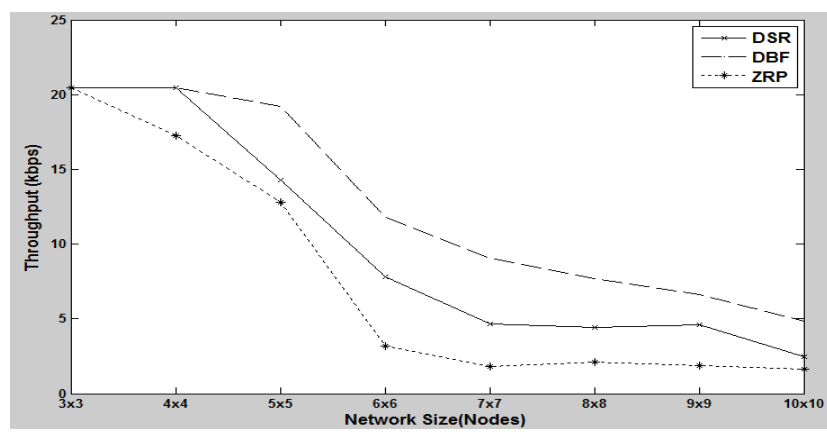

Fig 7: Average per flow throughput in square lattice network as a function of network size and routing protocol for 64 bytes packets

Our first observation is that network size and chain length, also mentioned in section 2.1, plays an important role in network performance. When network size and consequently chain length increases, there is a dramatic decrease in per flow throughput. The reason of this behavior is node interference [12], which increases by network size. RTS/CTS handshake cannot eliminate interference caused by hidden nodes, leading to a decrease in networks capacity. Interference range is greater than transmission range, meaning that an interfering signal can cause performance degradation even if its power is less than the power of transmission signal. If we could present analytically simulation results of each single chain, we would notice that in every case, two chains, the one at the top and the other at the bottom of the network perform better than the intermediate ones, justifying that node interference affects network performance.

Another observation is that the three routing protocols have similar behavior regardless of packet size. In all three cases DBF performs better, with ZRP having relatively inferior performance than the other two. A node using DBF forwards its packets through the shortest path, in this case a horizontal chain of nodes. Moreover, because nodes are static there are no invalid routes; packets are correctly forwarded to their destination, making a proactive routing protocol efficient in a square lattice network.

\subsection{Simulations in Lattice Networks}

In this section we examine two different topologies, both consisting of 18 nodes with a $200 \mathrm{~m}$ distance between them. The difference between the two scenarios is node placement. In the first case, nodes are placed in three chains consisting of six nodes, whereas in the second configuration we use six chains with three nodes each. The rest of the simulation parameters are the same as in section 2.2.Average per flow throughput values are shown in fig 8 .

Solid lines represent the average per flow throughput on a $3 \times 6$ network configuration whereas discontinuous line 
presents the respective of a $6 \times 3$ network configuration. Due to the smaller number of nodes per chain in the second case, interference level is decreased, leading to a more effective use of the common medium. DSR benefits from low interference level and due to reactive policy, has the best performance among the three routing protocols.

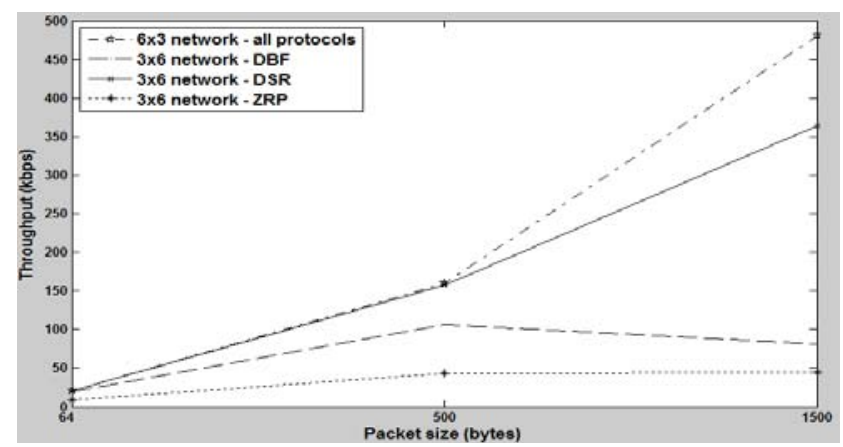

Fig 8: Average per flow throughput as a function of packet size and routing protocol.

As for the network that consists of 6 chains of three nodes each, its performance is shown by the discontinuous line in fig. 8. We used only one line, because all routing protocols have exactly the same performance. The number of nodes is the same as in the previous case, however the way nodes are placed in the network plays a significant role on networks' performance. Overall interference is much smaller than in the previous case, therefore performance is not affected by it. As a result all routing protocols perform exactly the same way, regardless the packet size.

\section{Lattice Networks with Limited Mobility}

3 In order to examine node mobility effects, in this section we present simulation results on two different topologies. In both cases, we assume a lattice network of 36 users, similar to the one in fig.4, distributed in a $1000 \times 1000 \mathrm{~m}$ area where nodes have $100 \mathrm{~m}$ distance from their neighbors. The left and right columns of nodes in this network are static, serving as source and destination nodes, respectively. We simulated two different scenarios. In the first one, apart from the static nodes at the edges, there is another static column of nodes, which is the $4^{\text {th }}$ from the left. In the second scenario, the two columns in the middle of the network $\left(3^{\text {rd }}\right.$ and $\left.4^{\text {th }}\right)$ are static.

In both scenarios simulation time is $600 \mathrm{sec}$. Nodes follow a random waypoint mobility pattern, with a maximum speed of $10 \mathrm{~m} / \mathrm{s}$ and $30 \mathrm{sec}$ pause time. All nodes use $11 \mathrm{Mbps} 802.11$ Distributed Coordination Function CSMA/CA as the MAC Layer protocol. In each scenario nodes use one of the DBF, DSR and ZRP routing protocols. There are 6 Constant bit rate (CBR) traffic source-destination pairs for every routing protocol, using
64,500 or 1500 bytes packets, sending at a constant rate of 40 packets $/ \mathrm{sec}$. We run a total number of 18 simulations scenarios with Table 2 showing the simulation parameters.

Table 2: Simulation Parameters

\begin{tabular}{|l|r|}
\hline Protocols & DBF,DSR,ZRP \\
\hline Simulation time & $600 \mathrm{sec}$ \\
\hline Number of nodes & 36 \\
\hline Simulation area & $1000 \times 1000$ \\
\hline Mobility model & Random Waypoint \\
\hline Max speed & $10 \mathrm{~m} / \mathrm{s}$ \\
\hline Pause time & $30 \mathrm{sec}$ \\
\hline Traffic Type & Constant bit rate \\
\hline Packet Size & $64,500,1500$ bytes \\
\hline Offered load & 40 packets $/ \mathrm{sec}$ \\
\hline Number of connections & 6 \\
\hline
\end{tabular}

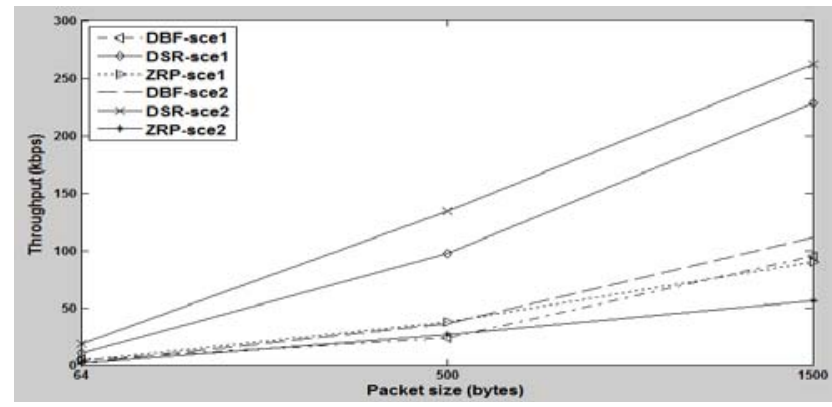

Fig.9: Average throughput per flow in limited mobility scenarios.

As shown in fig.9, all protocols attain lower throughput values when small packets are used. DBF and ZRP protocols have similar performance for the same topology and mobility configuration, whereas DSR outperforms them in every case.

Fig. 10 shows average delay per flow. In most of our simulations, when larger size packets are used, the is an increase in packet losses, resulting in lower delay values than those observed when small size packets are used.

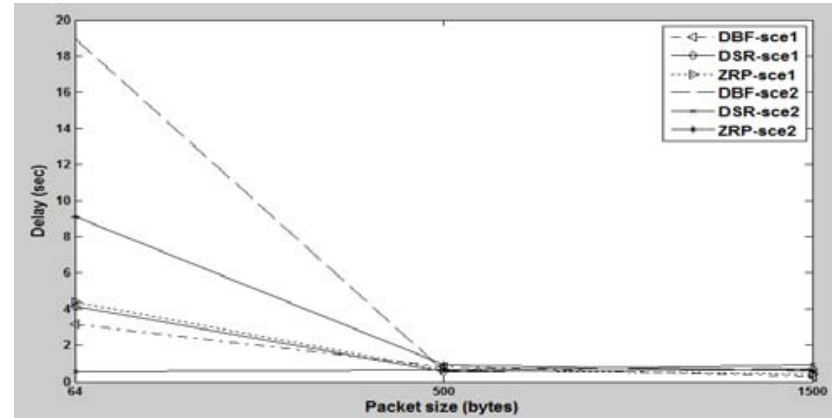

Fig.10: Average delay per flow as a function of packet size.

If we compare throughput results presented in figs 5 to 7 for a $6 \times 6$ lattice network topology with the results in fig. 9, we observe that there is an increase in throughput when 
mobility is introduced to the network in almost every case, irrespectively of the routing protocol used. First, we review results of Gupta and Kumar [13]. Node positions $\left\{\mathrm{X}_{\mathrm{i}}\right\}$ are independent and identically distributed and uniformly distributed in the disk of unit area, but fixed over time. The destination for each source node is a randomly chosen node in the network and the destinations are all chosen independently. The following results yield upper and lower bounds on the asymptotically feasible throughput. Main result 4 in [13] is that there exist constants $\mathrm{c}>0$ and $\mathrm{c}^{\prime}<+\infty$ such that

and

$$
\lim _{n \rightarrow \infty} \operatorname{Pr}\left\{\lambda(n)=\frac{c W}{\sqrt{n \log n}} \quad \text { is feasible }\right\}=1
$$

$$
\lim _{n \rightarrow \infty} \operatorname{Pr}\left\{\lambda(n)=\frac{c^{\prime} W}{\sqrt{n}} \quad \text { is feasible }\right\}=0
$$

where $n$ is the number of nodes per unit area, W is channel capacity and $\lambda(n)$ is the long term throughput.

Thus, within a factor of $\sqrt{n \log n}$, the throughput per Source-Destination (S-D) pair goes to zero like $\frac{R}{\sqrt{n}}$ in the case when the nodes are fixed. This result can be intuitively understood as follows. Every bit has to travel at least the distance that separates its source from its destination. It may travel this distance either through a single direct transmission or through multiple transmissions via relay nodes.

Assume for simplicity that all transmitting nodes transmit at the same power $P$. Let us focus on the transmission from a node $i$ to a node $j$. It can be seen that transmission from $i$ to $j$ will be unsuccessful whenever there is another transmitting interferer $k$ with distance $\mid X_{k}-X_{j} \leq(\beta / L)^{1 / \alpha} X_{i}$ $-\mathrm{X}_{\mathrm{j}} \mid$. In other words, there cannot be another sender in a disk of radius proportional to the transmission distance $\mathrm{X}_{\mathrm{i}}$ $-\mathrm{X}_{\mathrm{j}}$. Hence, a (successful) transmission over a distance $d$ incurs a cost proportional to $d^{2}$ by excluding other transmissions in the vicinity of the sender $i$. In order to maximize the transport capacity of the network, i.e., the total number of meters traveled by all bits per time unit, it is therefore beneficial to schedule a large number of short transmissions. Restricting transmissions to neighbors within a typical distance $O\left(\frac{1}{\sqrt{n}}\right)$ is the best we can do. Transport capacity is then at most $\sqrt{n}$ bits $\mathrm{m} / \mathrm{s}$. As there are $n$ sessions, each with an expected distance of $\Theta(1)$, the throughput per session can at best be $\mathrm{O}\left(\frac{1}{\sqrt{n}}\right)$.

In [14] theorem III-4 proves that it is possible to schedule $\Theta(\mathrm{n})$ concurrent successful transmissions per time slot with local communication. The question is how to forward packets between sources and destinations in order to use these transmissions, which can be achieved by spreading the traffic stream between the source and the destination to a large number of intermediate relay nodes. Each packet goes through one relay node that temporarily buffers the packet until final delivery to the destination is possible. For a source-destination pair S-D, all the other $n$ -2 nodes can serve as relay nodes. The goal is that in steady-state, the packets of every source node will be distributed across all the nodes in the network, hence ensuring that every node in the network will have packets buffered destined to every other node (except itself). This ensures that a scheduled sender-receiver pair always has a packet to send, in contrast to the case of direct transmission.

The question in [14] is how many times a packet has to be relayed in order to spread traffic uniformly to all nodes. In fact, as node location processes $\left\{\mathrm{X}_{\mathrm{i}}(\mathrm{t})\right\}$ are independent, stationary, and ergodic, it is actually sufficient to relay only once. This is because the probability for an arbitrary node to be scheduled to receive a packet from a source node $\mathrm{S}$ is equal for all nodes and independent of $\mathrm{S}$. Each packet then makes two hops, one from the source to its random relay node and one from that relay node to the destination. As no packet is transmitted more than twice, the achievable total throughput is $\Theta(n)$.

In order to prove that mobility increases capacity, they first exhibit a scheduling policy $\pi$ to select random senderreceiver pairs in each time slot $t$, such that all pairs can successfully transmit in time slot $t$. Then they use this policy as a building block to achieve throughput $\Theta(1)$ per S-D pair for large n. $\Theta(1)$ means that $\lambda(n)=C W$, independent of $\mathrm{n}$.

The theoretic estimation in [14] agrees with the simulation results presented in this paper. When we introduce mobility to the network, capacity increases in most cases. There is also an increase in average per flow throughput compared to the case where all nodes are static, irrespectively of the routing protocol used.

In our paper we examine the case of a network consisting of both static and mobile nodes. Suppose the total number of nodes in the network is $n$, and a portion of $m$ nodes are static, whereas the rest $n-m$ nodes follow a random waypoint mobility pattern. In this case, static nodes' throughput will be $\lambda(\mathrm{m})=\frac{C W}{(1+\Delta)^{2} \sqrt{m \operatorname{logm}}}$, whereas mobile nodes' throughput will be $\lambda(n-m)=C W$. Therefore total throughput is $\lambda(n)=\lambda(m)+\lambda(n-m)=C W\left(1+\frac{1}{(1+\Delta)^{2} \sqrt{m \operatorname{logm}}}\right)$ when the network consists of both static and mobile nodes. Also in this case, as proved in [13] there is a decrease in throughput as $m \rightarrow \infty$, in which case the ' 1 ' factor in the 
parenthesis is omitted. Moreover, as the number of static nodes $\mathrm{m}$ decreases, there is an increase in throughput, which conforms to the results of [14].

In many cases simulation results agree with the previous theoretic analysis for a network consisting of both static and mobile nodes. However there are cases where there is a difference between theoretical and simulation results. This is expected, as we simulate only a limited number of wireless networks. Moreover, throughput equations in [13] and [14] are approximate, meaning that they do not take into account the differences between routing protocols, or the effect of packets' size to the network.

\section{Random Topology Networks}

In this section, we introduce full node mobility and look into the comparative performance of the three routing algorithms in a random topology $11 \mathrm{Mbps}$ IEEE 802.11 b Ad Hoc network. Simulation area is $1000 \times 1000 \mathrm{~m}$ and the network consists of 30 users. The network operates in 802.11 Distributed Coordination Function CSMA/CA mode as before. We simulate CBR applications with the same parameters as in section 3 with flows' destinations chosen randomly from a uniform distribution. Simulation time is $600 \mathrm{sec}$. We use a RWP mobility model, with a maximum speed of $10 \mathrm{~m} / \mathrm{s}$ and $30 \mathrm{sec}$ pause time.

We simulate two different scenarios. In the first, each node acts exclusively either as a sender or a receiver of packets, therefore there are 15 active CBR flows. In the second scenario there are $30 \mathrm{CBR}$ flows since a node functions both as sender and receiver of packets whereas in both scenarios every node can act as a relay node. We use three different routing protocols, DBF, DSR and ZRP in each scenario and packets of 64, 500 and 1500 bytes long as in section 3. Nodes send with a rate of 40 packets/sec. The results in fig. 11 and 12 pertain to throughput and average delay respectively, for both scenarios.

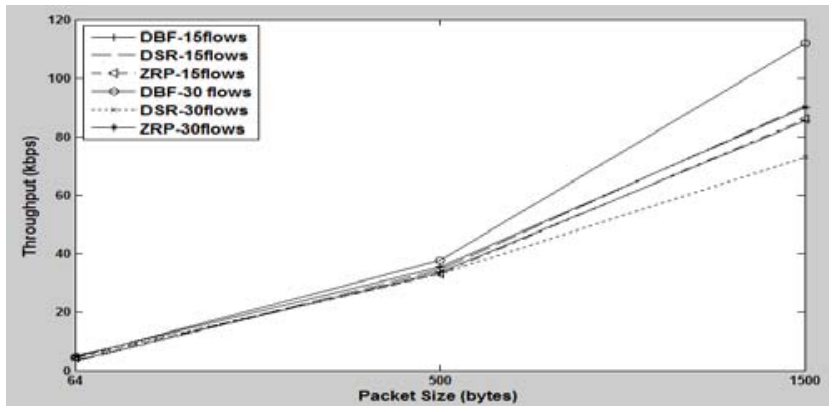

Fig.11: Average throughput per flow in a random topology configuration.

As seen in fig. 11, routing protocols exhibit similar performance in almost every case, with DSR performing slightly better when 500 and 1500 bytes packets are used.

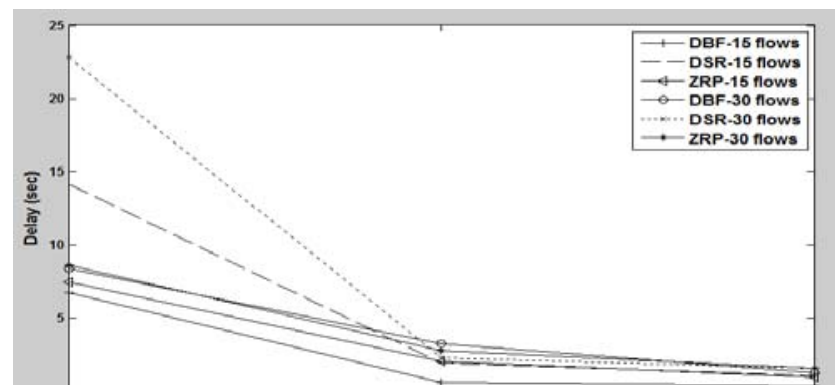

Fig.12: Average delay per flow as a function of the packet size.

In fig.12 DSR exhibits the largest delay in every case, especially when small packets are transferred. However this is counterbalanced by increased throughput (fig.11).

For the examined network configurations, we observe that DBF [15], which is a proactive protocol, achieved greater throughput than DSR and ZRP in the lattice network scenario. Its performance was average in the $3 \times 6$ scenario and the same as the performance of DSR and ZRP in the $6 \times 3$ scenario. Its performance deteriorates when we introduce node mobility to the network. When a node using DBF as routing protocol is disconnected from the network, a large number of interactions are needed between nodes for the disconnected node to be found. Another disadvantage is that routing information is forwarded at specific moments. When nodes are mobile, updates are very frequent, due to changes in topology. As a result, a large amount of the available throughput is consumed for the transmission of this information, depriving network capacity for data transmission. These issues would be more visible if node density was lower, i.e. the same number of nodes was distributed in a larger size area. In such a case, nodes would disconnect more frequently from the network, resulting in a larger amount of interactions and updates, and consequently in decreased throughput and increased delay values.

DSR [16] performs better than DBF and ZRP when we introduce node mobility. This routing protocol stores routes, therefore a source node maintains information about the path followed during route discovery. Route discovery is achieved by sending RREQ packets, through which a source node will learn all intermediate nodes through which information will travel towards its destination. The destination node responds with a RREP packet, so it will learn all possible paths to one destination. This process is followed by all nodes and can lead to increased overhead (therefore decreased throughput), especially in large size networks. However, when routes become invalid due to node's mobility, the source node will continue to forward wrong routing information; consequently all nodes will have false routing information. 
In terms of delay, DSR shows greater delay in the simulated scenario of section 4 , where all nodes move and the offered load is increased compared to the scenarios of section 3. This shows that DSR is sensitive to network load and mobility conditions.

When the ZRP routing protocol is used, its performance is slightly better than the performance of DBF in many cases. As mentioned before, ZRP defines zones whose radius is the maximum number of neighbor users. In this zone, IARP [17] (Intrazone Routing Protocol) protocol is used, making route requests easier without examining all nodes in the network. The amount of unused routing information is also decreased. Distant nodes can be accessed through reactive routing, using IERP [17] (Interzone Routing Protocol) protocol. ZRP's advantage is that local topology is known. This way when there is an unstable connection, packets are forwarded through an alternative path. Moreover, this can be used to reduce path length, in case the distance between two nodes is reduced. This explains the slight difference in performance compared to DBF. Most nodes are relatively close to one another, implying that in many cases proactive routing is used, similar to DBF. Reactive routing is used for distant nodesdestinations, leading to increased throughput in this case. Due to node mobility, network topology must be rediscovered many times, which leads to an increase in delay, due to topology information exchange between nodes. Choosing zone radius is a tradeoff between routing efficiency and control information in order for the zone to become known. In our simulations, we choose a zone radius which systematically decreases the amount of required control information.

Similar behavior, though improved in terms of throughput and packet losses, is observed when an FTP application is used (instead of CBR) in the simulated scenarios described. Another important aspect is that in most cases where mobility is introduced, we observe large delays when small packets are transferred. We conducted several simulations in order to explain this behavior. Our first conclusions are that in this case, buffer size plays significant role in delay. By decreasing a node's buffer size, there is a significant decrease in delay, and in some cases, an increase in throughput is observed. Analytical results are presented in section 5 .

Regarding packet losses, in the course of a packet's transmission, a source node counts the numbers of short (ns) and long $\left(\mathrm{n}_{\mathrm{l}}\right)$ retries. Let a source node transfer a DATA frame with a packet of length equal to or less than the RTS threshold P, or an RTS frame. If a correct ACK or CTS frame, respectively, is received within timeout limits, then the $\mathrm{ns}_{\mathrm{s}}$-counter is zeroed; otherwise $\mathrm{ns}_{\mathrm{s}}$ is advanced by one. Similarly, the $\mathrm{n}_{1}$-counter is zeroed or advanced by one in case of reception or absence of a correct ACK frame (within timeout) confirming the successful transfer of a DATA frame with a packet of length greater than P. When any of $n_{s}$ and $n_{1}$ attains its limit $N_{s}$ or $N_{1}$ respectively, the current packet is rejected. After the rejection or success of a packet transmission, $\mathrm{n}_{\mathrm{r}}, \mathrm{n}_{\mathrm{s}}$, and $\mathrm{n}_{1}$ values are zeroed [18]. Limits defined by IEEE $802.11 \mathrm{~b}$ are 7 and 4 for $\mathrm{n}_{\mathrm{s}}$ and $\mathrm{n}_{1}$ respectively. We used these values in our simulations. However we do not present packet loss results in this paper, due to page limitations, and will be presented in a future extended work.

\section{Simulations Altering Buffer Size}

In section 2.2 we conducted simulations on lattice networks which consist of chains of nodes. We present throughput results showing that as the number of nodes of a chain increases, therefore network size increases, there is a decrease in average per flow throughput which leads to stabilization of throughput value.

We have not yet presented however, results about the delay of these simulations. As expected, we observe an increase in delay as chain size increases, taking values even in the case where chain length is short, e.g. 4 or 5 nodes. Fig. 15 shows delay for the networks described in section 2.2 for 1500 bytes packets.

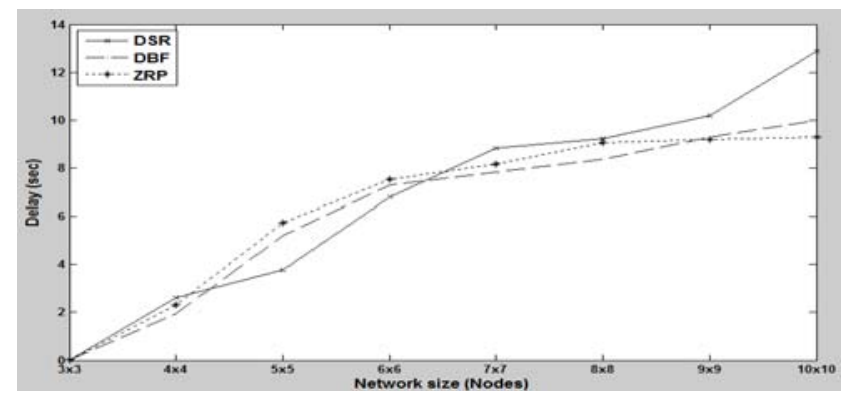

Fig.13: Average per flow delay in square lattice network, as a function of network size and routing protocol for 1500 bytes packets

Figures 14 and 15 show delay results for 500 and 64 bytes packets respectively.

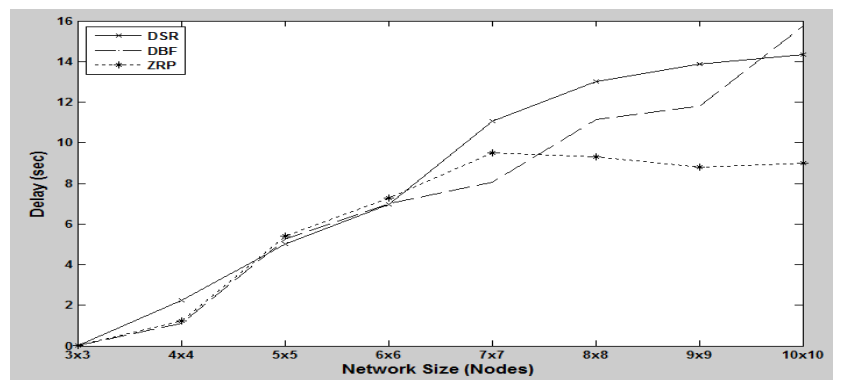

Fig.14: Average per flow delay in square lattice network, as a function of network size and routing protocol for 500 bytes packets 
As shown by figures 13-15, average delay value increases for each network size as the size of transferred packets decreases, especially when network size is greater than 6x6. However the most remarkable observation is that in those cases delay gets greater values when small packets are transferred through the network, e.g. 64 bytes packets, rather than when large size packets, e.g. 1500 bytes packets are transferred.

This behavior can be explained through an analysis of buffer size. In our simulations we used a buffer of 50000 bytes and FIFO queuing scheme is used. When 64 bytes packets are used, a larger amount of packets can be stored in buffer, compared to the case when 1500 bytes packets are transferred through the network. Therefore in the first case of 64 bytes packets, a greater amount of time is required in order for those packets to be stored, processed and forwarded to the next hop.

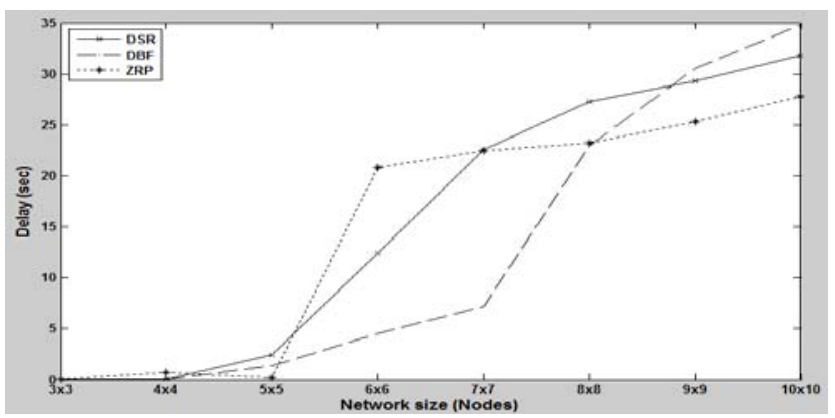

Fig.15: Average per flow delay in square lattice network, as a function of network size and routing protocol for 64 bytes packets

When network size is small, as in the cases of $3 \times 3$ and $4 \times 4$ as shown in figures $13-15$, the total amount of packets is decreased compared to the rest of the cases. Due to the decreased amount of packets in the network it is harder for buffers to get fully loaded, therefore delay is considerably decreased. However, in the rest cases when the total amount of packets is increased, more packets are stored in a nodes' buffer leading to very large delay values. Of course there are differences in these values which depend on the routing protocol used; however there is no dispute that all simulated routing protocols follow this behavior.

In order to examine the validity of these results in relation to packet size, we conducted the same simulation using 280 bytes and 1000 bytes packets. The results of these simulations show that all routing protocols follow similar behavior to the one observed in figures 13 to 15 .

Our first conclusion is that delay increases when small size packets are transferred through the network and it decreases as packets of larger size are used. We made the assumption that this behavior happens due to the number of packets stored in buffer. When small packets are transferred a greater amount of packets are stored in buffer rather than in the case of large size packets leading to an increase in delay. In order to check if our speculation is correct, we conduct similar simulations to the ones presented in section 2.2. The only difference in this case is that we change buffer size. Until now we used a buffer size of 50000 bytes, whereas now we change buffer size. Buffer size depends on the size of packets we use. Figures 16 and 17 present simulation results for 64 bytes packets for 5000,500 and 128 bytes buffer size respectively.

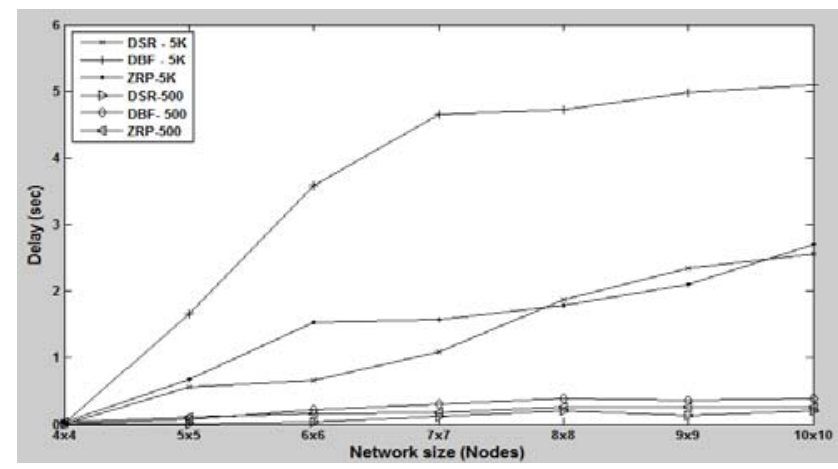

Fig.16: Average per flow delay in square lattice network, with horizontal flows, as a function of network size and routing protocol for 64 bytes packets for 5000 and 500 bytes buffer size.

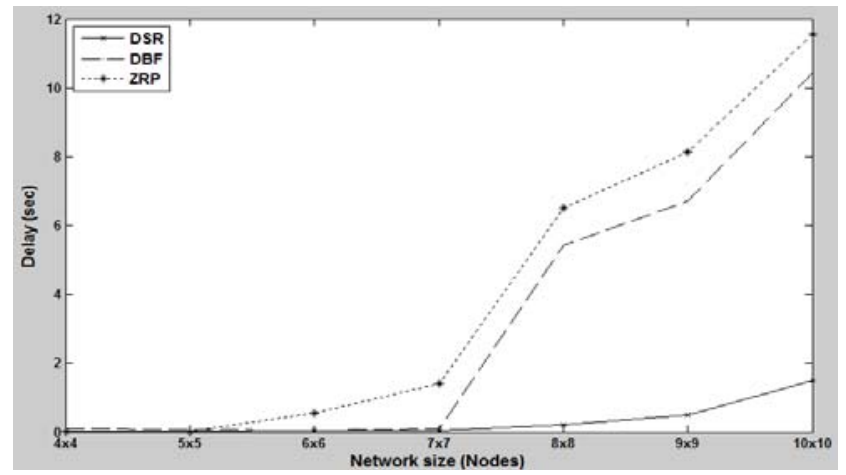

Fig.17: Average per flow delay in square lattice network, with horizontal flows, as a function of network size and routing protocol for 64 bytes packets and 128 bytes buffer size.

As shown in fig. 16 and 17 there is a decrease in delay as buffer size decreases, especially when buffer size is 5000 and 500 bytes. In the last case, for 128 bytes buffer size, ZRP and DBF appear to have increased delay compared to DSR protocol, however even in this case delay is severely decreased compared to the case of 50000 bytes buffer.

For further confirmation of our results we present similar results for 500 and 1500 bytes packets in the following figures. In every case, there is a decrease in delay when a smaller size buffer is used. In the case of 500 bytes packets we used 1500 and 500 buffer size, while when 1500 bytes 
packets are transferred our simulations where conducted using 5000 and 3000 bytes buffers.

It is more than obvious that when buffer size is decreased there is a severe decrease in average per flow delay, especially when network size is increased. In our simulations this is clearly observed when network size is $6 \times 6$ or $7 \times 7$.

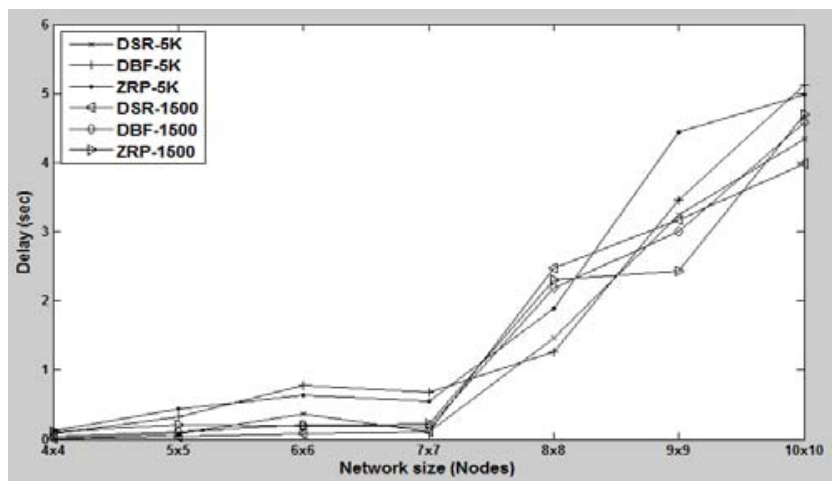

Fig.18: Average per flow delay in square lattice network, with horizontal flows, as a function of network size and routing protocol for 500 bytes packets and 5000 and 1500 bytes buffer size.

Of course this conclusion is not very safe when buffer size is such that only an extremely limited number of packets can be stored in, as in the case presented in fig 17. In this case, packets of 64 bytes are transferred through the network, and buffer size is 128 bytes. Therefore, only 2 packets can be stored in a node's buffer. In this case, when DSR and DBF routing protocols are used delay is almost equal to the delay presented in fig 6 for the respective routing protocols. However when the routing protocol is ZRP, delay is almost 10 to 12 times increased compared to the other routing protocols, even for networks of average size, showing that routing protocol has an important role in network's performance. However, limiting buffer size still appears to be an effective way of decreasing delay.

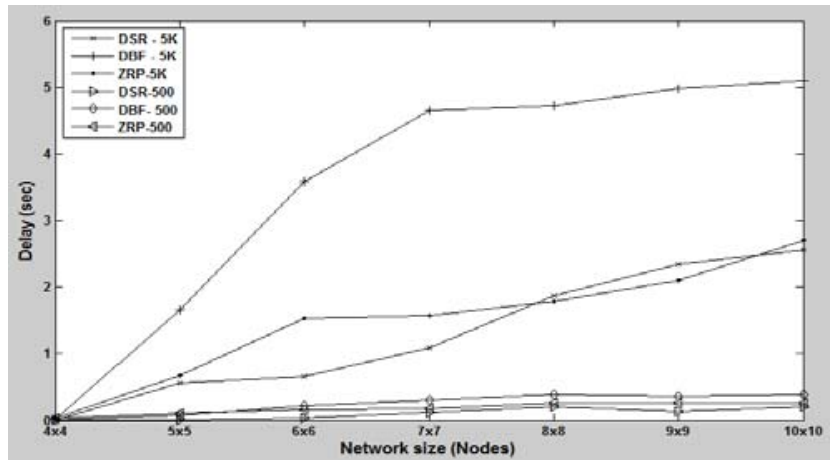

Fig.19: Average per flow delay in square lattice network, with horizontal flows, as a function of network size and routing protocol for 500 bytes packets for 5000 and 1500 bytes buffer size.
Another aspect which needs to be examined is the impact buffer decrease has on throughput. When buffer size is decreased, the maximum amount of packets a buffer can store is decreased. Simulation results already presented, show that such a decrease is beneficial in terms of delay. However by decreasing buffer's capacity, the possibility of packets to be dropped is increased, leading to throughput deterioration, although we managed to improve delay by decreasing it.

Fig. 20 presents throughput simulation results when 1500 bytes packets are transferred through the network.

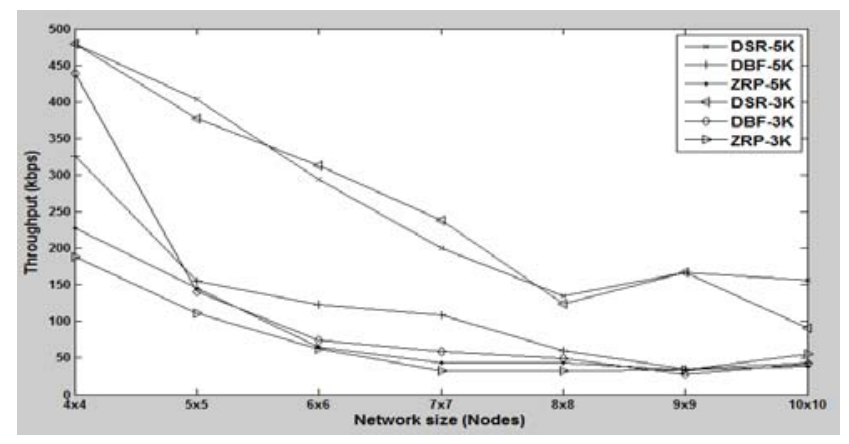

Fig 20: Average per flow throughput in square lattice network, with horizontal flows, as a function of network size and routing protocol for 1500 bytes packets, for 5000 and 1500 bytes buffer size.

Those results compared to the simulation results in fig. 6 , show that in most cases there is an increase in per flow throughput as buffer size decreases. Especially minimum throughput values are considerably improved when smaller size buffer is used. Our conclusion is that when smaller size buffer is used there is a decrease in delay without an impact in throughput. Contrarily in most cases there is an increase in throughput, which leads to an overall improvement in a network's performance.

\section{Conclusions}

Our focus in this paper is to evaluate the performance of an Ad Hoc network, in scenarios involving both static and mobile nodes, using different routing protocols and offered load conditions. We compare three different routing protocols, each representing one of the three types of routing protocols, i.e., proactive, reactive and hybrid. Our main contribution (relative to previous work) is the systematic analysis of these routing protocols in a variety of network topologies including static nodes scenarios, scenarios with limited node mobility and full node mobility (sections 2, 3, 4 respectively), citing a simple throughput theoretical analysis for each of those topologies. 
Our first observation is that, per flow throughput is affected by the way nodes are placed in the network. Moreover, a node's and a network's performance is affected by node mobility and the choice of routing protocols. We showed that in a network configuration where all nodes are mobile and there is an increased traffic load to be transmitted, per node throughput is increased when a reactive routing protocol is employed, especially when larger data segments are transmitted.

In terms of comparative performance evaluation, we show advantages of reactive routing protocols such as DSR, leading to increased throughput achieved when nodes are mobile, at the expense of increased delay. The efficiency in route discovery contributes to increased delay in this case. As for proactive and hybrid routing protocols, DBF and ZRP respectively, there seems to be relatively small difference between them. ZRP shows some advantages compared to DBF when nodes are mobile in which its proactive routing component performs better than reactive routing. However DBF is more effective in the case of static chains of nodes or in square lattice networks.

Moreover we examined the effect of buffer size in both static and mobile lattice networks' performance. In our simulations, reducing buffer size causes delay reduction and throughput improvement for all routing protocols. Especially when DSR and DBF routing protocols are used there is an increase in throughput even for small size networks, while in the case of ZRP routing protocol, throughput decreases slightly for small size networks when buffer size decreases. However even in the case of ZRP, while the size of buffer decreases there is an increase in throughput as network size grows bigger.

\section{References}

[1] M. Abolhasan, T. Wysocki, "A review of routing protocols for mobile ad hoc networks",_Ad Hoc Networks, Vol. 2, Issue 1, pp 1-22, 1 January 2004.

[2] R. Misra, C. R. Mandal, "Performance comparison of AODV/DSR on-demand routing protocols for ad hoc networks in constrained situation”, IEEE Int. Conf. on Personal Wireless Communications, pp. 86- 89, Jan. 2005.

[3] G. Jayakumar, G. Ganapathy, "Performance Comparison of Mobile Ad-hoc Network Routing Protocol", Int. J. of Comp. Science and Net. Security, Vol.7, No.11, Nov. 2007.

[4] H. Jiang and J. J. Garcia-Luna-Aceves, "Performance comparison of three routing protocols for ad hoc networks", Proceeding of IEEE ICCCN 2001.

[5] P. Johansson, T. Larsson, N. Hedman, B. Mielczarek, and M. Degermark, "Scenario-Based Performance Analysis of Routing Protocols for Mobile Ad Hoc Networks", 5th ACM/IEEE Int. Conf. on Mobile computing and networking, pp. 195-206. 1999.

[6] J. Broch, D. A. Maltz, D. B. Johnson, Y. Hu, J. Jetcheva, "A Performance Comparison of Multi-Hop Wireless Ad Hoc Network Routing Protocols", Proc. of the 4th ACM/IEEE Int. conf. on Mobile computing and networking, pp. 85-97. 1998.

[7] Li Layuan, Li Chunlin, Yaun Peiyan, "Performance evaluation and simulations of routing protocols in ad hoc networks", Computer Communications, Vol. 30 .pp 1890-1898. 2007
[8] Azzedine Boukerche, "Performance Evaluation of Routing Protocols for Ad Hoc Wireless Networks", Journal of Mobile Networks and Ap. Vol. 9, No 4. pp.333-342, 2004.

[9] Qualnet, www.scalable-networks.com

[10] I.F. Akyildiz, Xudong Wang, "A survey on wireless mesh networks”, IEEE Communications Magazine, Vol. 43, Issue 9, pp. S23 S30, Sept. 2005

[11] J. Li, C. Blake Douglas,S. J. De Couto, H. I. Lee, R. Morris, "Capacity of Ad Hoc Wireless Networks", 7th annual international conference on Mobile computing and networking, 2001.

[12] Kaixin Xu, Mario Gerla, Sang Bae . "How Effective is the IEEE 802.11 RTS/CTS Handshake in Ad Hoc Networks?", Proceeding of GLOBECOM'02.

[13] P. Gupta, P. R. Kumar, "The capacity of wireless networks," IEEE Transactions on Information Theory, vol. 46, no. 2, Mar 2000

[14]_M. Grossglauser, D. N. C. Tse, "Mobility increases the capacity of Ad Hoc wireless networks", IEEE/ACM Transactions on Networking, Vol. 10, No. 4, 2002.

[15] D. Bertsekas,R. Gallaher, "Data Networks", Second Edition, Prentice Hall,1992.

[16] J . Wu, "An Extended Dynamic Source Routing Scheme in Ad Hoc Wireless Networks",HICSS, 2002.

[17] Z. Haas, M. Pearlman, P. Samar, "The Zone Routing Protocol (ZRP) for Ad Hoc Networks," IETF InternetDraft,draft-ietf-manet-zone-zrp04.txt, July 2002.

[18] Andrey Lyakhov, Florian Simatos "Hybrid RTS/CTS mechanism in Wi-Fi Ad Hoc Networks with Correlated Channel Failures", 17th IMACS, 2005

Evaggelos C. Chatzistavros received his diploma of Computer and Electrical Engineer from Democritus University oh Thrace, Greece, in 2007, has completed his M. Sc thesis on "Performance evaluation of routing protocols in IEEE Ad Hoc networks" from the Department of Electrical and Computer Engineering, Democritus University of Thrace, in 2010 and is currently working on his Ph. D thesis. His research interests are communication networks, wireless networks and performance evaluation of routing protocols.

George Stamatelos is an assistant professor of Electrical and Computer Engineering in Democritus University of Thrace. He received his Ph.D. in EECS from Concordia University, Montreal, Canada in 1992 and has since held various positions in both the academia and industry. He teaches courses in Telecommunications and Computer Networks and his research interests include stochastic processes, queuing theory, communication networks and nomadic computing. 